JOURNAL CLUB

\title{
Overdiagnosis of pulmonary emboli by CT pulmonary angiograms
}

The authors of this article suggest that pulmonary emboli (PEs) are being overdiagnosed by high-resolution CT pulmonary angiography (CTPA), which detect small, isolated subsegmental PEs that are not clinically important. Data from the USA show an $80 \%$ rise in the incidence of PEs between 1998 and 2006 after CTPA was introduced. Despite the large increase in diagnosis, age-adjusted mortality from PE (deaths in the US population) has changed little: from 12.3 to 11.9 per 100000 .

Presently the best evidence for overdiagnosis comes from a trial that randomised 1417 patients with suspected PE to receive either ventilation-perfusion (VQ) scanning or CTPA. Although CTPA detected more emboli than VQ scans $(19.2 \%$ vs $14.2 \%)$, there was no difference in mortality $(0.3 \%$ vs $0.3 \%)$ over three months. The main harms from overdiagnosis are unnecessary treatment with anticoagulation and exposure to contrast and radiation. There has been a substantial increase in complications arising from anticoagulation that parallels the increased incidence of PE.

There are limitations to inferring overdiagnosis from epidemiological data and currently it is impossible to say which emboli are not clinically important. At present clinicians should test more selectively and consider that the harms of treating isolated subsegmental PEs may outweigh the benefits. An ongoing prospective cohort study assessing the safety of withholding treatment for stable patients with isolated subsegmental PE may shed further light on this issue.

- Wiener RS, Schwartz LM, Woloshin S. When a test is too good: how CT pulmonary angiograms find pulmonary emboli that do not need to be found. BMJ 2013;347:f3368.

Uruj Hoda

Correspondence to Dr Uruj Hoda, Department of Respiratory Medicine, Barnet Hospital, Wellhouse Lane, EN5 3DJ, UK; uruj@doctors.org.uk

Competing interests None.

Provenance and peer review Not commissioned; internally peer reviewed.

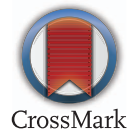

To cite Hoda U. Thorax 2014;69:925.

Published Online First 21 October 2013

Thorax 2014;69:925. doi:10.1136/thoraxjnl-2013-204663 